\title{
INSURANCE DRAWDOWN-TYPE CONTRACTS FOR A PHASE-TYPE RISK PROCESS PERTURBED BY BROWNIAN MOTION ${ }^{1}$
}

ŚLĄSKI

PRZEGLĄD

STATYSTYCZNY

Nr 15(21)

\author{
Zbigniew Palmowski \\ Wrocław University of Science and Technology \\ Joanna Tumilewicz \\ University of Wrocław \\ e-mails: zbigniew.palmowski@gmail.com; joanna.tumilewicz@gmail.com
}

DOI: $10.15611 /$ sps.2017.15.10

JEL Classification: C61, G01, G13, G22

\begin{abstract}
Summary: In this paper we consider the insurance polices based on drawdown and drawup events where an underlying asset is derived by a classical risk process with phasetype claim sizes perturbed by Brownian motion. The drawdown/drawup process we define as a difference between the historical maximum/minimum and current asset value. We consider four contracts presented in [Palmowski, Tumilewicz 2016]. The first one is an insurance contract where the protection buyer is paying a constant premium with intensity $p$ until the drawdown of fixed size occurs. In return he/she receives a certain insured amount at the drawdown epoch. The second insurance contract may expire early if a certain fixed drawup event occurs prior to a fixed drawdown. The last two contracts are extensions of the previous ones by an additional cancellable feature which allows an investor to terminate the contract earlier. We focus here on an extensive numerical analysis when claim sizes are phase-type.
\end{abstract}

Keywords: insurance contract, fair valuation, drawdown, Lévy process, drawup, optimal stopping, Brownian motion.

\section{Introduction}

The drawdown of a given process is the distance of the current value away from the maximum value it has attained to date. Similarly, the drawup of a given process is defined as the current rise of the present value over the running minimum. Both of them have been customarily used as dynamic risk measures. In fact, drawdown processes do not only provide dynamic measures of risk but can also be viewed as measures of relative regret. Similarly, the drawup process can be viewed as a measure

${ }^{1}$ This work is partially supported by the National Science Centre under the grant 2015/17/B/ST1/01102 (2016-2019). 
of relative satisfaction. Thus, a drawdown or a drawup of a certain number of units may signal the time in which an investor may choose to change his/her investment position depending on his/her perception of future moves of the market and his/her risk aversion.

The interest in asset drawdowns has been significantly raised by the recent financial crisis. Therefore the risk managment of drawdowns has become very important among practitioners; see e.g. [Grossman, Zhou 1993] for porfolio optimization under constraints on the drawdown process, [Carr et al. 2011], [Magdon-Ismail, Atiya 2004] for the distribution of the maximum drawdown of drifted Brownian motion and the time-adjusted measure of performance known as the Calmar ratio and [Pospisil, Vecer 2010; Vecer 2006; 2007] for drawdown processes as dynamic measures of risk. For an overview of the existing techniques for the analysis of market crashes, as well as a collection of empirical studies of the drawdown process and the maximum drawdown process, please refer to [Sornette 2003]. It is then natural that fund managers have a strong incentive to seek insurance against drawdowns.

In this paper we follow [Palmowski, Tumilewicz 2016] and [Zhang et al. 2013] pricing some insurance contracts against drawdown (and drawup) events and identifying optimal stopping rules. We also identify for them the so-called fair premium rates when contracts' prices equal zero. In its simplest form, the first drawdown insurance contract involves a continuous premium payment paid by the investor (protection buyer) to insure a drawdown of an underlying asset value to a pre-specified level. Possibly, the buyer of the insurance contract may think that it is unlikely to get a large drawdown and he/she might want to stop paying the premium. Therefore we expand the simplest contract by adding a cancellable feature. In this case, the investor receives the right to terminate the contract earlier and in this case he/she pays a penalty for doing so. We show that the investor's optimal cancellable time is based on the first passage time of the drawdown process.

Moreover, we also consider a related insurance contract that protects the investor from a drawdown preceding a drawup. In other words, the insurance contract expires early if a drawup event occurs prior to a drawdown. From the investor's perspective, when a drawup is realized there is little need to insure against a drawdown. Therefore this drawup contingency automatically stops the premium payment and is an attractive feature that will potentially reduce the cost of drawdown insurance. Finally, we also added a cancellable feature to this contract. 
In this paper we start from presenting the latest results derived by [Palmowski, Tumilewicz 2016] giving very general formulas for the prices of the mentioned above insurance contracts and identifying the STATYSTYCZNY optimal stopping rules. The main goal of this paper is to give a more statistical, and hence more practical, analysis of these formulas. We suggest here some new algorithms of finding all quantities when claim sizes (or jumps of asset prices) have so-called phase-distributions.

Phase-type distributions are the computational vehicle of much of modern applied probability. Typically, if a problem can be solved explicitly when the relevant distributions are exponentials, then the problem may admit an algorithmic solution involving a reasonable degree of computational effort if one allows for the more general assumption of phase-type structure. We refer to the book by [Asmussen 2003] for an overview of the properties of the phase-type distributions.

The paper is organized as follows. In Section 2 we present insurance contacts that we will work with and identify their prices and the optimal stopping rules. In Section 3 deals with the numerical analysis. We complete our paper with our conclusions in Section 4.

\section{Drawdown-type contracts}

We work on complete filtered probability space $(\Omega, F, \mathbf{P})$ satisfying the usual conditions. We model a logarithm of risky underlying asset price $\log S_{t}$ by a Cramér-Lundberg risk process perturbed by an independent Brownian motion $B_{t}$ :

$$
X_{t}=x+d t+\sigma B_{t}-\sum_{i=1}^{N_{t}} \eta_{i} .
$$

Above $N_{t}$ is a Poisson process with intensity $\beta>0$ and $\left\{\eta_{i}\right\}$ is a independent sequence of i.i.d. jumps. This is a very natural choice for modelling the asset process and this choice is due to a better fit of the evolution of the stock price process to the real data. Indeed in recent years the empirical study of financial data reveals the fact that the distribution of the log-return of stock price exhibits features which cannot be captured by the normal distribution, such as heavy tails and asymmetry. For the purpose of replicating more effectively these features and for reproducing a wide variety of implied volatility skews and smiles, there has been a general shift in the literature to modelling stock price with exponential jump-diffusion process as an alternative 
to the exponential of a linear Brownian motion; see [Oksendal, Sulem 2004] for overviews. Note also that process $X_{t}$ given in (1) is a particular case of so-called spectrally negative Lévy process that is, in fact, our price process $S_{t}=\exp \left\{X_{t}\right\}$ is a geometric Lévy process.

What is more, we assume that the distribution of jumps of $X_{t}$ given in (1) is phase-type. A distribution $F_{\eta}$ of $\eta$ is said to be of phasetype (and we will write $\eta \approx \mathrm{Ph}(\gamma, \mathbf{T})$ ) if $\eta$ has the same distribution as the lifetime of some terminating the Markov process starting from the vector $\gamma$, having finitely many states $N$ and having time homogeneous transition rates with subintensity $\mathbf{T}$. Then

$$
F_{\eta}(\phi)=1-\gamma \exp \{\mathbf{T} \phi\} \mathbf{1},
$$

for $\phi \geq 0$, where $\mathbf{1}$ is a vector of ones. This gives also the form of the density $f_{\gamma}(\phi)$ of $\gamma$ :

where

$$
f_{\gamma}(\phi)=\gamma \exp \{\mathbf{T} \phi\} \mathbf{t},
$$

$$
\mathbf{t}=-\mathbf{T} \mathbf{1} .
$$

Most of the well-known distributions are of phase-type:

- exponential distribution with intensity $\rho$

- $\quad$ in this case $N=1, \mathbf{T}=-\rho$ and $\gamma=1$;

- Erlang distribution $\operatorname{Erl}(\rho, N)$ :

$$
f_{\gamma}(\phi)=\rho^{N} \frac{\phi^{N-1}}{(N-1) !} e^{-\rho \phi}
$$

- in this case $\gamma=(1,0, \ldots, 0)$ and

$$
\mathbf{T}=\left(\begin{array}{ccccc}
-\rho & \rho & 0 & \cdots & 0 \\
0 & -\rho & \rho & \cdots & 0 \\
\vdots & \vdots & \vdots & \ddots & \vdots \\
0 & 0 & 0 & \cdots & -\rho
\end{array}\right)
$$

- hyperexponential distribution:

$$
f_{\gamma}(\phi)=\sum_{i=1}^{N} \gamma_{i} \rho_{i} e^{\rho_{i} \phi}
$$

- in this case:

$$
\mathbf{T}=\left(\begin{array}{ccccc}
-\rho_{1} & 0 & 0 & \cdots & 0 \\
0 & -\rho_{2} & 0 & \cdots & 0 \\
\vdots & \vdots & \vdots & \ddots & \vdots \\
0 & 0 & 0 & \cdots & -\rho_{N}
\end{array}\right)
$$


- a mixture of Erlang distributions with the same scale parameter $\rho>0$ :

$$
f_{\gamma}(\phi)=e^{\rho \phi} \sum_{i=1}^{L} \gamma_{i} \frac{\rho^{i}}{(i-1) !} \phi^{i-1}
$$

- in this case $N=\frac{L(L+1)}{2}$ and the subintensity matrix $\mathbf{T}$ is a matrix made of blocks of increasing size from 1 to $L$ of type (3) located along the diagonal.

Many identities for the spectrally negative Lévy process $X$ presented in (1) is given in terms of so-called scale functions which are defined in the following way. For $r \geq 0$ we define a continuous and strictly increasing function $W^{(r)}$ on $[0, \infty)$ with the Laplace transform given by:

$$
\int_{0}^{\infty} e^{\phi u} W^{(r)}(u) d u=\frac{1}{\psi(\phi)-r}
$$

where

$$
\psi(\phi)=\log \mathbb{E}\left[e^{\phi X_{1}}\right]
$$

is a Laplace exponent of $X_{t}$. This is the first scale function. The second one is related with the first one via the following relationship:

$$
Z^{(r)}(u)=1+r \int_{0}^{u} W^{(r)}(\phi) d \phi .
$$

The Laplace exponent $\psi$ defined in (5) calculated for the process $X$ given in (1) equals:

$$
\psi(\phi)=d \phi+\frac{\sigma^{2} \phi^{2}}{2}+\beta\left(\gamma(\phi \mathbf{I}-\mathbf{T})^{-1} \mathbf{t}-1\right),
$$

where $\mathbf{t}$ is given in (2) and $\mathbf{I}$ is an identity matrix of appropriate size $N$.

Using the definition of the scale function $W^{(r)}$ given in (4) and [6, Prop. 5.4], we can conclude the following result of [4, Prop. 2.1]. Let $I$ be a set of (the sign-changed) negative roots:

$$
I:=\left\{\varphi_{i}: \psi\left(-\varphi_{i}\right)=r \text { and } \operatorname{Re}\left(\varphi_{i}>0\right)\right\}
$$

and

$$
\Phi(r):=\sup \{\varphi \geq 0: \psi(\varphi)=r\}
$$


If elements in $I$ are distinct, then for $r \geq 0$ the scale function is $\mathrm{Nr}$ 15(21) written as:

where

$$
W^{(r)}(u)=\frac{e^{\Phi(r)}}{\psi^{\prime}(\Phi(r))}-\sum_{i=1}^{N-1} B_{i} e^{-\varphi_{i} u}
$$

$$
B_{i}:=-\frac{1}{\psi^{\prime}\left(-\varphi_{i}\right)} .
$$

Later we will focus on the numerical work for two distributions of the generic jump $\eta$ :

- Erlang distribution $\operatorname{Erl}(2, \rho)$ :

$$
f_{\gamma}(\phi)=\rho^{2} \phi e^{-\rho \phi}
$$

with Laplace exponent given by

$$
\psi(\phi)=d \phi+\frac{\sigma^{2} \phi^{2}}{2}+\beta\left(\frac{\rho}{\rho+\phi}\right)^{2}-\beta
$$

and

- hyperexponential distribution

$$
f_{\gamma}(\phi)=\gamma_{1} \rho_{1} e^{-\rho_{1} \phi}+\gamma_{2} \rho_{2} e^{-\rho_{2} \phi}
$$

for a probability vector $\left(\gamma_{1}, \gamma_{2}\right)$ and Laplace exponent

$$
\psi(\phi)=d \phi+\frac{\sigma^{2} \phi^{2}}{2}+\beta\left(\gamma_{1} \frac{\rho_{1}}{\rho_{1}+\phi}+\gamma_{2} \frac{\rho_{2}}{\rho_{2}+\phi}\right)-\beta
$$

For these distributions we will evaluate all the insurance contracts described before. In Table 1 and Table 2 below are presented the roots of $\psi(\varphi)-r=0$ necessary to evaluate $W^{(r)}$ in (8).

Table 1. The roots of $\psi(\varphi)-r=0$ for process $X$ with parameters $d=0.05, \sigma=0.4$, $\beta=0.1$ and jumps with Erlang distribution $\operatorname{Erl}(2,4)$. The free interest rate $r=0.01$

\begin{tabular}{|c|c|c|c|}
\hline$\Phi(r)$ & $\varphi_{1}$ & $\varphi_{2}$ & $\varphi_{3}$ \\
\hline 0.321 & $4.318-1.075 i$ & 0.315 & $4.318+1.075$ \\
\hline
\end{tabular}

Source: own work.

Table 2. The roots of $\psi(\varphi)-r=0$ for process $X$ with parameters $d=0.05, \sigma=0.4$, $\beta=0.1$ and jumps with hyperexponential distribution $\gamma_{1}=0.4, \gamma_{2}=0.6, \rho_{1}=3$, $\rho_{2}=4$. The free interest rate $r=0.01$

\begin{tabular}{|c|c|c|c|}
\hline$\Phi(r)$ & $\varphi_{1}$ & $\varphi_{2}$ & $\varphi_{3}$ \\
\hline 0.236 & 0.476 & 3.149 & 4.236 \\
\hline
\end{tabular}

Source: own work. 
For the cases presented in Table 1 and Table 2 we can explicitely calculate the scale function $W^{(r)}$. For Erlang distribution we have that

$$
\begin{aligned}
& W^{(r)}(\phi)=16.199 e^{0.321 \phi}-0.646 \cos (1.075 \phi) e^{-4.318 \phi} \\
& -15.552 e^{-0.315 \phi}-0,-0.361 \sin (1.075 \phi) e^{-4.318 \phi}
\end{aligned}
$$

and for hyperexponential distribution we get

$$
\begin{gathered}
W^{(r)}(\phi)=15.889 e^{0.236 \phi}-15.529 e^{-0.476 \phi}-0.161 e^{-3.149 \phi} \\
-0.199 e^{-4.236 \phi}
\end{gathered}
$$

Let us denote by:

$$
\bar{X}_{t}=\sup _{\mathrm{s} \leq \mathrm{t}} X_{s}, \underline{X}_{t}=\inf _{\mathrm{s} \leq \mathrm{t}} X_{s},
$$

the maximum and minimum logarithm of the asset price within time interval $[0, t]$. In this paper, we analyze insurance contracts related with the drawdown and drawup processes. The drawdown is the difference between running the maximum of the process and its current value and the the drawup is the difference between the process' current value and its running minimum. Here, we additionally allow that the drawdown and drawup processes start from some points $y$ and $z$, respectively. That is,

$$
D_{t}=\bar{X}_{t} \vee y-X_{t}, U_{t}=X_{t}-\underline{X}_{t} \wedge(-z) \text {. }
$$

Above, the values $y$ and $-z$ can be interpreted as the historical maximum and historical minimum of process $X$. Crucial for further work are the following first passage times of the drawdown process and drawup processes, respectively:

$$
\begin{aligned}
& \tau_{D}^{+}(a)=\inf \left\{t \geq 0: D_{t} \geq a\right\}, \tau_{D}^{-}(a)=\inf \left\{t \geq 0: D_{t} \leq a\right\} \\
& \tau_{U}^{+}(a)=\inf \left\{t \geq 0: U_{t} \geq a\right\}, \tau_{U}^{-}(a)=\inf \left\{t \geq 0: U_{t} \leq a\right\} .
\end{aligned}
$$

Later we will use the following notational convention:

$$
\begin{gathered}
\mathbf{P}_{\mid y}[\cdot]:=\mathbf{P}\left[\cdot \mid D_{0}=y\right], \\
\mathbf{P}_{|y| z}[\cdot]:=\mathbf{P}\left[\cdot \mid D_{0}=y, U_{0}=z\right], \\
\mathbf{P}_{x|y| z}[\cdot]:=\mathbf{P}\left[\cdot \mid X_{0}=x, D_{0}=y, U_{0}=z\right] .
\end{gathered}
$$

Finally, we denote $\mathbf{P}_{x}[\cdot]:=\mathbf{P}\left[\cdot \mid X_{0}=x\right]$ with $\mathbf{P}:=\mathbf{P}_{\mathbf{0}}$ and $\mathbb{E}_{\mid y}, \mathbb{E}_{|y| z}, \mathbb{E}_{x|y| z}, \mathbb{E}_{x}, \mathbb{E}$ will be the corresponding expectations to the above measures. We also used the following notational convention: $\mathbb{E}\left[\cdot \mathbb{1}_{\{A\}}\right]=\mathbb{E}[\cdot ; \mathrm{A}]$ for any event $A$. 


\subsection{Drawdown insurance contract}

Nr 15(21) In this subsection we consider the insurance contract in which the protection buyer will pay constant premium $p>0$ continuously until the drawdown of size $a>0$ occurs. In return he/she receives the insured amount of $\alpha>0$ at the drawdown moment. Let $r>0$ be the risk-free interest rate. The contract price is equal to the discounted value of the future cash-flows:

$$
f(y, p)=\mathbb{E}_{\mid y}\left[-\int_{0}^{\tau_{D}^{+}(a)} e^{-r t} p \mathrm{~d} t+\alpha e^{-r \tau_{D}^{+}(a)}\right] .
$$

In this contract the investor wants to protect him/herself from the asset price $S_{t}=e^{X_{t}}$ falling from the previous maximum more than fixed level $e^{a}$ for some $a>0$. In other words, he/she believes that even if the price will go up again after the first drawdown of size $e^{a}$ it will not bring him/her sufficient profit. Therefore he/she is ready to take this type of contract to reduce loss by getting $\alpha>0$ at the drawdown epoch.

The next result gives the price of the contract $f(y, p)$. The proof of all theoretical results can be found in [Palmowski, Tumilewicz 2016] and we skipped them here focusing later only on the numerical and statistical analysis.

Theorem 1. The value of the contract (16) is by

$$
f(y, p)=\left(\frac{p}{r}+\alpha\right)\left(Z^{(r)}(a-y)-r W^{(r)}(a-y) \frac{W^{(r)}(a)}{W^{\prime(r)}(a)}\right)-\frac{p}{r} .
$$

A fair situation for both sides, the insurance company and investor, is when the contract price equals 0 . We say then that the premium $p^{*}$ is fair when

$$
f\left(y, p^{*}\right)=0 .
$$

From Theorem 1 we can derive the following theorem.

Theorem 2. For the contract (16) a fair premium equals:

$$
p^{*}=\frac{r \alpha\left(Z^{(r)}(a) W^{\prime(r)}(a)-r\left(W^{(r)}(a-y)\right)^{2}\right)}{W^{\prime(r)}(a)-Z^{(r)}(a) W^{\prime(r)}(a)+r\left(W^{(r)}(a-y)\right)^{2}} .
$$




\subsection{Cancellable feature}

We now extend the previous contract by a cancellable feature. In other words, we give the investor the right to terminate contract payment at the termination time fixed fee $c>0$ for doing it anytime prior to a pre-specified drawdown of size $a>0$. This contract is interesting for the investor who is not willing to pay a premium any longer when he/she stops believing that a large drawdown may happen. The contract value equals then:

$$
\begin{aligned}
F(y, p)=\sup _{\tau \in \mathrm{T}}\left[-\int_{0}^{\tau_{D}^{+}(a) \wedge \tau} e^{-r t} p \mathrm{~d} t-c e^{-r t} \mathbb{1}_{\left\{\tau<\tau_{D}^{+}(a)\right\}}\right. \\
\left.+\alpha e^{-r \tau_{D}^{+}(a)} \mathbb{1}_{\left\{\tau_{D}^{+}(a)<\tau\right\}}\right]
\end{aligned}
$$

where $\mathrm{T}$ is a family of all $\mathrm{F}_{t}$-stopping times.

One of the main goals of this paper is to identify the optimal stopping rule $\tau^{*}$ that realizes the price $F(y, p)$. To do this, we use the "guess and verify" approach. This means that we first guess the candidate stopping rule and then verify if this is truly the optimal stopping rule using the well-known Verification Lemma (see [Kyprianou 2006]). Our guess will be that the optimal stopping rule is the first passage time of the drawdown process below some level $\theta$, that is that

$$
\tau^{*}=\tau_{D}^{-}\left(\theta^{*}\right) \in \mathrm{T}
$$

for some $\theta^{*} \in(0, a)$. We chose this class of stopping times since it represents the opposite situation to the insured one. The contract insures against a huge drawdown, so when the drawdown process is falling the moment of reward payment is receding. Obviously we choose the optimal level $\theta^{*}$ such that it maximizes the value function

$$
F(y, p, \theta):=F(y, p)
$$

calculated under the assumption that $\tau_{D}^{-}(\theta)$ realizes the supremum in (19). Thus

$$
\theta^{*}=\inf \left\{\theta \in(0, a): \frac{\partial}{\partial \theta} F(y, p, \theta)=0 \text { and } \forall \varsigma \geq 0 F(y, p, \varsigma) \leq F(y, p, \theta)\right\} \text {. }
$$

Observe now that the value of the basic contract without a cancellable feature, $f(y, p)$ given in (16) is an increasing function with respect to $y$. So the value of the basic contract $f$ is smallest at 
point 0 . Let us consider a situation when $f(0, p)>-c$. This means that the smallest expected value that an investor can receive from the basic contract is greater than the fee he/she will pay in the case of terminating insurance earlier. When this is the case then the optimal stopping strategy for the investor is to never terminate the contract, that is $\tau=\infty$. To eliminate this trivial case we assume from now that

$$
f(0, p) \leq-c
$$

which is equivalent to saying that

$$
\frac{p}{r}-c>\left(\frac{p}{r}+\alpha\right)\left(Z^{(r)}(a)-r \frac{\left(W^{(r)}(a)\right)^{2}}{W^{\prime(r)}(a)}\right) \geq 0 .
$$

For the stopping rule (20) we can explicitly compute the value of the cancellable drawdown contract $F(y, p, \theta)$ given in (21). For $y>\theta$ we get

$$
\begin{gathered}
F(y, p, \theta)=\left(\frac{p}{r}+\alpha\right) Z^{(r)}(a-y)+\left(\frac{p}{r}-c\right) \frac{W^{(r)}(a-y)}{W^{(r)}(a-\theta)} \\
-\left(\frac{p}{r}+\alpha\right) Z^{(r)}(a-\theta) \frac{W^{(r)}(a-y)}{W^{(r)}(a-\theta)}-\frac{p}{r} .
\end{gathered}
$$

Note that if $y \leq \theta$, then the investor will terminate the contract immediately:

$$
F(y, p, \theta)=-c .
$$

In fact, if $y \leq \theta^{*}$ the investor should not buy the contracts since it is optimal to terminate at its beginning. This implies that we should only consider contracts for which $y>\theta^{*}$.

We can verify that (20) indeed holds true (see Theorem 3 of [Palmowski, Tumilewicz 2016] for details).

Theorem 3. Assume that (22) holds. If $\theta^{*} \in(0, y)$, then the stopping time $\tau_{D}^{-}\left(\theta^{*}\right)$ is an optimal stopping rule for the stopping problem (19).

\subsection{Incorporating drawup contingency}

The investor might like to buy a contract which has some maturity conditions. This means that the contract will end when these conditions are fulfilled. Therefore in this paper we will also consider an insurance contract which provides protection from any specified drawdown with a certain drawup contingency. In particular, this contract may expire earlier 
if a fixed drawup event occurs prior to the drawdown of the same level. Choosing the drawup event is very natural since it corresponds to some market upward trend and therefore the investor might want to stop paying the premium when this event happens. Under a risk-neutral measure the value of this contract equals:

$$
k(y, z, p):=\mathbb{E}_{|y| z}\left[-\int_{0}^{\tau_{D}^{+}(a) \wedge \tau_{U}^{+}(a)} e^{-r t} p \mathrm{~d} t+\alpha e^{-r \tau_{D}^{+}(a)} \mathbb{1}_{\left\{\tau_{D}^{+}(a)<\tau_{U}^{+}(a)\right.}\right],
$$

for $a>0$. At the beginning we will find this value function and then identify the fair premium $p^{*}$ under which

$$
k\left(y, z, p^{*}\right)=0 .
$$

Note that

$$
k(y, z, p)=\left(\frac{p}{r}+\alpha\right) v(y, z)+\frac{p}{r} \lambda(y, z)-\frac{p}{r},
$$

where

$$
\begin{aligned}
& v(y, z):=\mathbb{E}_{|y| z}\left[e^{-r \tau_{D}^{+}(a)} ; \tau_{D}^{+}(a)<\tau_{U}^{+}(b)\right], \\
& \lambda(y, z):=\mathbb{E}_{|y| z}\left[e^{-r \tau_{U}^{+}(a)} ; \tau_{U}^{+}(b)<\tau_{D}^{+}(a)\right] .
\end{aligned}
$$

According to [Palmowski, Tumilewicz 2016], if $a \leq y+z$, then the following holds

$$
\begin{gathered}
\lambda(y, z)=\frac{W^{(r)}(a-y)}{W^{(r)}(2 a-y-z)}, \\
v(y, z)=Z^{(r)}(a-y)-Z^{(r)}(2 a-y-z) \frac{W^{(r)}(a-y)}{W^{(r)}(2 a-y-z)} .
\end{gathered}
$$

When $a>y+z$, then we have

$$
\lambda(y, z)=\frac{W^{(r)}(a-y)}{W^{(r)}(2 a-y-z)}-\frac{1}{r} \frac{W^{\prime(r)}(a)}{\left(W^{(r)}(a)\right)^{2}}\left(Z^{(r)}(a-y)-Z^{(r)}(z)\right)
$$

and

$$
\begin{gathered}
v(y, z)=Z^{(r)}(z)-Z^{(r)}(a) \frac{W^{(r)}(a-y)}{W^{(r)}(a)} \\
+\frac{1}{r} Z^{(r)}(a) \frac{W^{\prime(r)}(a)}{\left(W^{(r)}(a)\right)^{2}}\left(Z^{(r)}(a-y)-Z^{(r)}(z)\right) .
\end{gathered}
$$

Now we can state the following result of [Palmowski, Tumilewicz 2016]. 
Theorem 4. The price of the contract defined in (25) is given in (27) with $v(y, z)$ and $\lambda(y, z)$ given in (28)-(29) and (30)-(31).

From (27) if follows the following theorem.

Theorem 5. For the contract (25) the fair premium defined in (26) equals:

$$
p^{*}=\frac{r \alpha v(y, z)}{1-v(y, z)-\lambda(y, z)} .
$$

\subsection{Cancellable feature}

We will also consider now the additional possibility of the termination of the previous contract. In this contract the protection buyer can terminate his/her position by paying fee $c>0$. The value of this contract equals then

$$
\begin{aligned}
& K(y, z, p):=\sup _{\tau \in \mathrm{T}}\left[-\int_{0}^{\tau_{D}^{+}(a) \wedge \tau_{U}^{+}(a) \wedge \tau} e^{-r t} p \mathrm{~d} t\right. \\
& \left.\quad+\alpha e^{-r \tau_{D}^{+}(a)} \mathbb{1}_{\left\{\tau_{D}^{+}(a)<\tau_{U}^{+}(a) \wedge \tau\right\}}-c e^{-r \tau} \mathbb{1}_{\left\{\tau<\tau_{D}^{+}(a) \wedge \tau_{U}^{+}(a)\right\}}\right] .
\end{aligned}
$$

Similarily to cancellable drawdown contract, we are looking for optimal stopping rule $\tau^{*}$ which realizes supremum of $K(y, z, p)$. We use the "guess and verify" approach again. The candidate for optimal strategy is given by

$$
\tau^{*}=\tau_{D}^{-}\left(\theta^{*}\right) \in \mathrm{T}
$$

for some $\theta^{*} \in(0, a)$. We chose the same family of stopping time since the intuition stays the same. The reward is paid when the drawdown is growing, so we still expect that the optimal stopping rule is denoted by the first moment when the drawdown is declining. The presence of additional maturity conditions is only going to affect the optimal stopping level $\theta^{*}$. We denote by

$$
K(y, z, p, \theta):=K(y, z, p)
$$

caluculated for stopping time of form (34). It is natural to look for $\theta$ which maximizes function $K(y, z, p, \theta)$ in order to prove the optimality of (33). We define $\theta^{*}$ as follows:

$$
\begin{array}{r}
\theta^{*}:=\inf \left\{\theta \in(0, a): \frac{\partial}{\partial \theta} K(y, z, p, \theta)=0 \text { and } \forall \varsigma\right. \\
\geq 0 K(y, z, p, \varsigma) \leq K(y, z, p, \theta)\} .
\end{array}
$$


At the beginning of analyzing this contract, note that if $k(y, z)>$ $-c$ it is optimal for the investor to never terminate the contract and hence $\tau=\infty$. Again this happens because the smallest expected value that investor can receive from the basic contract is greater than the fee which should be paid. To avoid this trivial case we will assume that there exists at least one point $\left(y_{0}, z_{0}\right)$ for which $k\left(y_{0}, z_{0}\right) \leq-c$. This assumption is equivalent to the following inequality:

$$
\frac{p}{r}-c>\left(\frac{p}{r}+\alpha\right) v\left(y_{0}, z_{0}\right)+\frac{p}{r} \lambda\left(y_{0}, z_{0}\right) \geq 0 .
$$

Similarly, we calculate the value of $K(y, z, p, \theta)$ for $\tau^{*}$ given in (34). For $y>\theta$ we have

$$
=\left\{\begin{array}{c}
K(y, z, p, \theta)= \\
k(y, z, p)-(k(\theta, a-\theta)+c) \frac{W^{(r)}(a-y)}{W^{(r)}(a-\theta)}+c_{a}\left(Z^{(r)}(a-y)-Z^{(r)}(z)\right) \\
\text { for } a>y+z \\
k(y, z, p)-(k(\theta, y+z-\theta)+c) \frac{W^{(r)}(a-y)}{W^{(r)}(a-\theta)} \\
\text { for } y+z-\theta<a \leq y+z \\
0 \text { for } a<y+z-\theta,
\end{array}\right.
$$

where

$$
c_{a}=\left(\frac{p}{r}+\alpha\right)\left(1-Z^{(r)}(a) \frac{W^{\prime(r)}(a)}{\left(W^{(r)}(a)\right)^{2}}\right)+\frac{p}{r} \frac{1}{r} \frac{W^{\prime(r)}(a)}{\left(W^{(r)}(a)\right)^{2}}
$$

and $k(y, z, p)$ is given by (27) with $v(y, z)$ and $\lambda(y, z)$ calculated in (28)-(29) for $a \leq y+z$ and (30)-(31) for $a>y+z$. Moreover, for $\theta \leq y$ we should stop the contract immediately, so

$$
K(y, z, p, \theta)=-c \text {. }
$$

The next main result given in Theorem 7 of [Palmowski, Tumilewcz 2016] gives the price of the above insurance contract.

Theorem 6. Assume that (36) holds. If $\theta^{*} \in(0, y)$, then $\tau_{D}^{-}\left(\theta^{*}\right)$ is the optimal stopping rule solution for (32).

\section{Numerical analysis}

In this section we analyze numerically all the insurance contracts and check their dependence on the chosen parameters of the model. We focus on the classical risk process perturbed by Brownian motion given in (1). The main contribution of this paper lies in the extensive numerical 
analysis for cases when the jumps $\eta_{i}$ of logarithm of the asset price have phase-type distrbutions. These distrubutions are widely used in financial and actuarial applications and have the huge potential of modelling all possible shapes of jump distributions. They are also very convenient from the point of view of parametrisation and calibration since we can choose a general number of parameters to estimate.

\subsection{Fair premium for drawdown insurance}

We start from pricing the drawdown contract $f(y, p)$ given in (16) using Theorem 1 and, from identifying the expression for the fair premium $p^{*}$ given in (18) when jumps have an Erlang distribution (10) using the scale function $W^{(r)}$ given in (14), see Figure 1 and Figure 2.

Note that by increasing starting drawdown $D_{0}=y$, one may rapidly increase the value of the fair premium $p^{*}$. This happens because when the starting position of drawdown is near to insured level $a$, then we have less to wait. The fair premium has to be higher since it must balance the reward and the premium payment period is shorter. In fact, if $\sigma>0$, then $p^{*}$ value tends to $\infty$ when $y \uparrow a$. This follows from the fact that in this case we have $W^{(r)}(0)=0$ and the denominator in the expression for the fair premium $p^{*}$ goes to 0 as $y \uparrow a$.

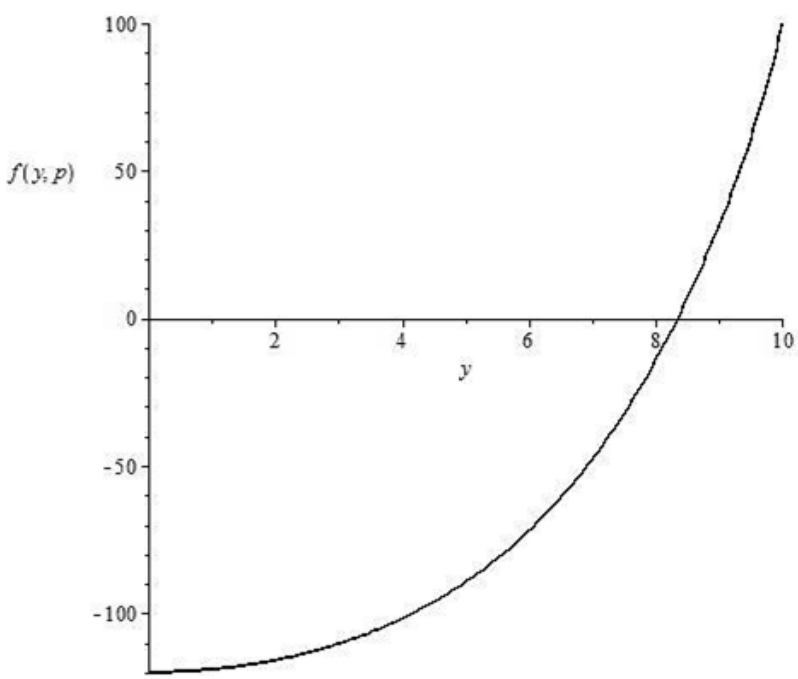

Fig. 1. The value of drawdown contract $f(y, p)$ with respect to the starting position of drawdown $y$, for process $X$ with parameters $d=0.05, \sigma=0.4, \beta=0.1$ and with jumps from Erlang distribution $\operatorname{Erl}(2,4)$. Parameters: free interest rate $r=0.01$, drawdown level $a=10$, reward $\alpha=100$, premium $p=1.4$

Source: own work. 


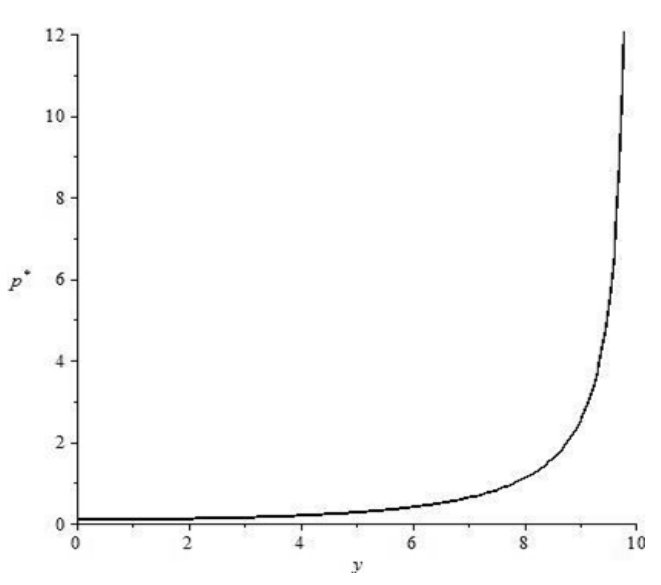

Fig. 2. Fair premium $p^{*}$ for drawdown contract $f(y, p)$ with respect to the starting position of drawdown $y$, for process $X$ with parameters $d=0.05, \sigma=0.4, \beta=0.1$ and with jumps from Erlang distribution $\operatorname{Erl}(2,4)$. Parameters: free interest rate $r=0.01$, drawdown level $a=10$, reward $\alpha=100$, premium $p=1.4$

Source: own work.

A similar result could be obtained for hyperexponential distribution (12) with scale function given in (15):

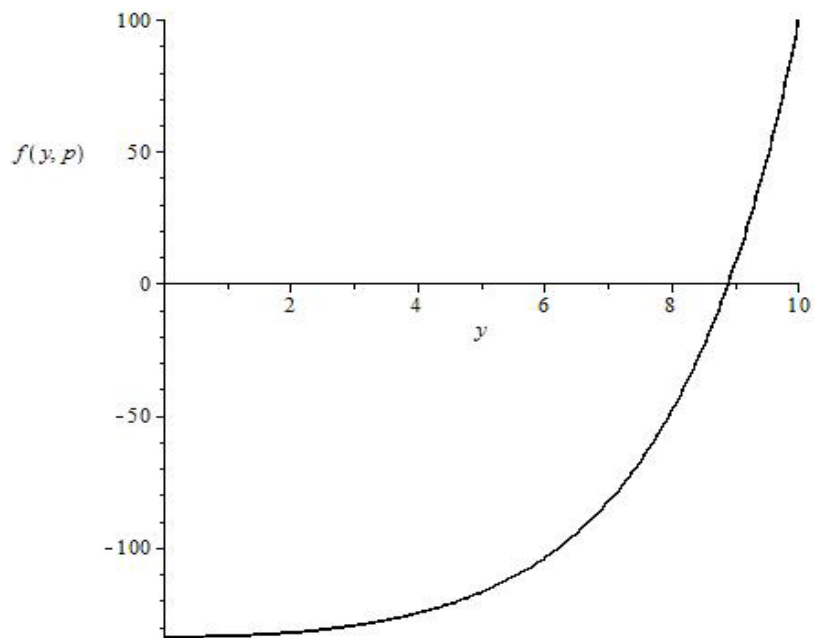

Fig. 3. The value of drawdown contract $f(y, p)$ with respect to the starting position of drawdown $y$, for process $X$ with parameters $d=0.05, \sigma=0.4, \quad \beta=0.1$ and with jumps from hyperexponential distribution $\gamma_{1}=0.4, \gamma_{2}=0.6, \rho_{1}=3, \rho_{2}=4$. Parameters: free interest rate $r=0.01$, drawdown level $a=10$, reward $\alpha=100$, premium $p=1.4$

Source: own work. 

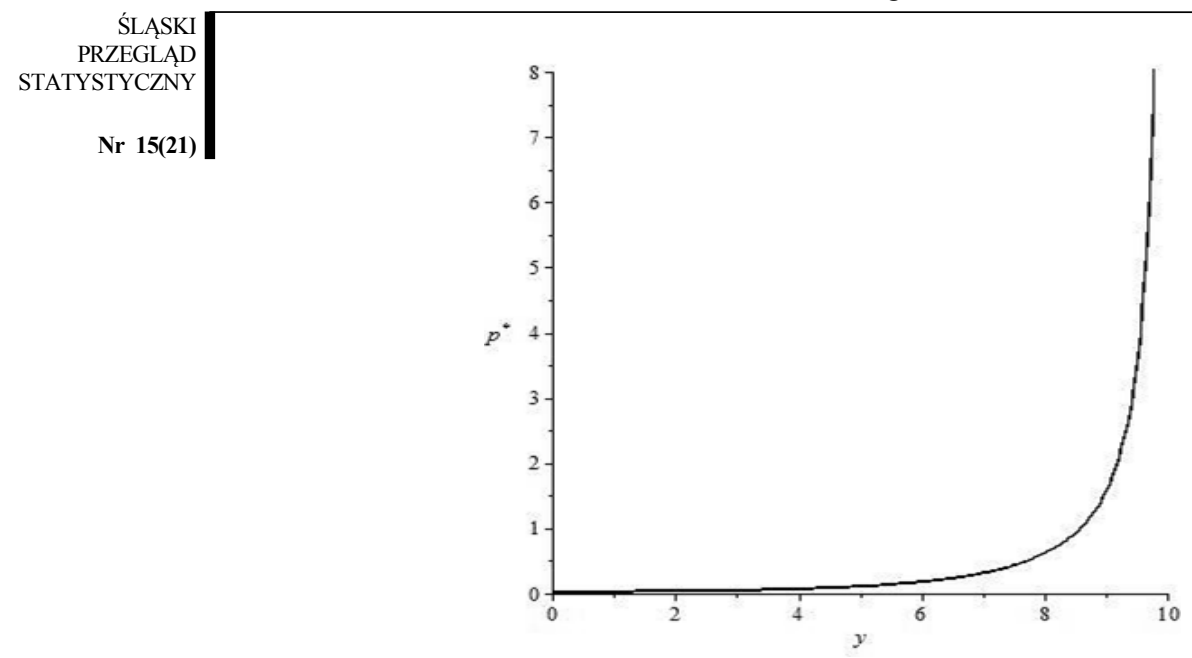

Fig. 4. Fair premium $p^{*}$ for drawdown contract $f(y, p)$ with respect to the starting position of drawdown $y$, for process $X$ with parameters $d=0.05, \sigma=0.4, \beta=0.1$ and with jumps from hyperexponential distribution $\gamma_{1}=0.4, \gamma_{2}=0.6, \rho_{1}=3, \rho_{2}=4$. Parameters: free interest rate $r=0.01$, drawdown level $a=10$, reward $\alpha=100$, premium $p=1.4$

Source: own work.

\subsection{Cancellable drawdown insurance}

We now price the contract $F(y, p)$ defined in (19) and identified in Theorem 3 and we also find the optimal stopping rule $\tau^{*}$ given in (20) for $\theta^{*}$ defined in (23). Because of the numerical work done in the previous subsection it suffices to find only function $g\left(y, p, \theta^{*}\right)$ and $\theta^{*}$.

Remember that from (24) for $\theta<y$ we have:

$$
\begin{gathered}
F(y, p, \theta)=\left(\frac{p}{r}+\alpha\right) Z^{(r)}(a-y)+\left(\frac{p}{r}-c\right) \frac{W^{(r)}(a-y)}{W^{(r)}(a-\theta)} \\
-\left(\frac{p}{r}+\alpha\right) Z^{(r)}(a-\theta) \frac{W^{(r)}(a-y)}{W^{(r)}(a-\theta)}-\frac{p}{r},
\end{gathered}
$$

where $c$ is the fee which the investors pay when he/she terminates the contract earlier, and for $\theta \geq y$

$$
F(y, p, \theta)=-c \text {. }
$$

Figure 5 and Figure 6 depict the dependence of the function $F(y, p, \theta)$ on $\theta$. The straight line at the end of the $F(y, p, \theta)$ follows from the fact that the function $F$ is constant equal to $-c$ for $\theta \geq y$. When $\theta^{*}$ is at the beginning of that straight line this means that the investor should not take this insurance contract. This means that we 
are looking for $\theta^{*}$ that maximizes function $F$ on $(0, y)$. Note also that by (24) and the definition of the optimal $\theta^{*}$ given in (20) the level $\theta^{*}$ does not depend on the starting position of the drawdown $D_{0}=y$ as long as $y>\theta^{*}$. In particular, for our set of parameters we have $\theta^{*} \approx 2.0$ for jumps with Erlang distribution and $\theta^{*} \approx 1.5$ for hyperexponential distribution.

Figure 5 and Figure 6 depict also the dependence of the function $F(y, p, \theta)$ on the premium rate $p$. Note that a smaller premium rate produces higher values of the insurance contract $F$ given in (19). It also affects the level of optimal $\theta^{*}$. By increasing premium $p$ we

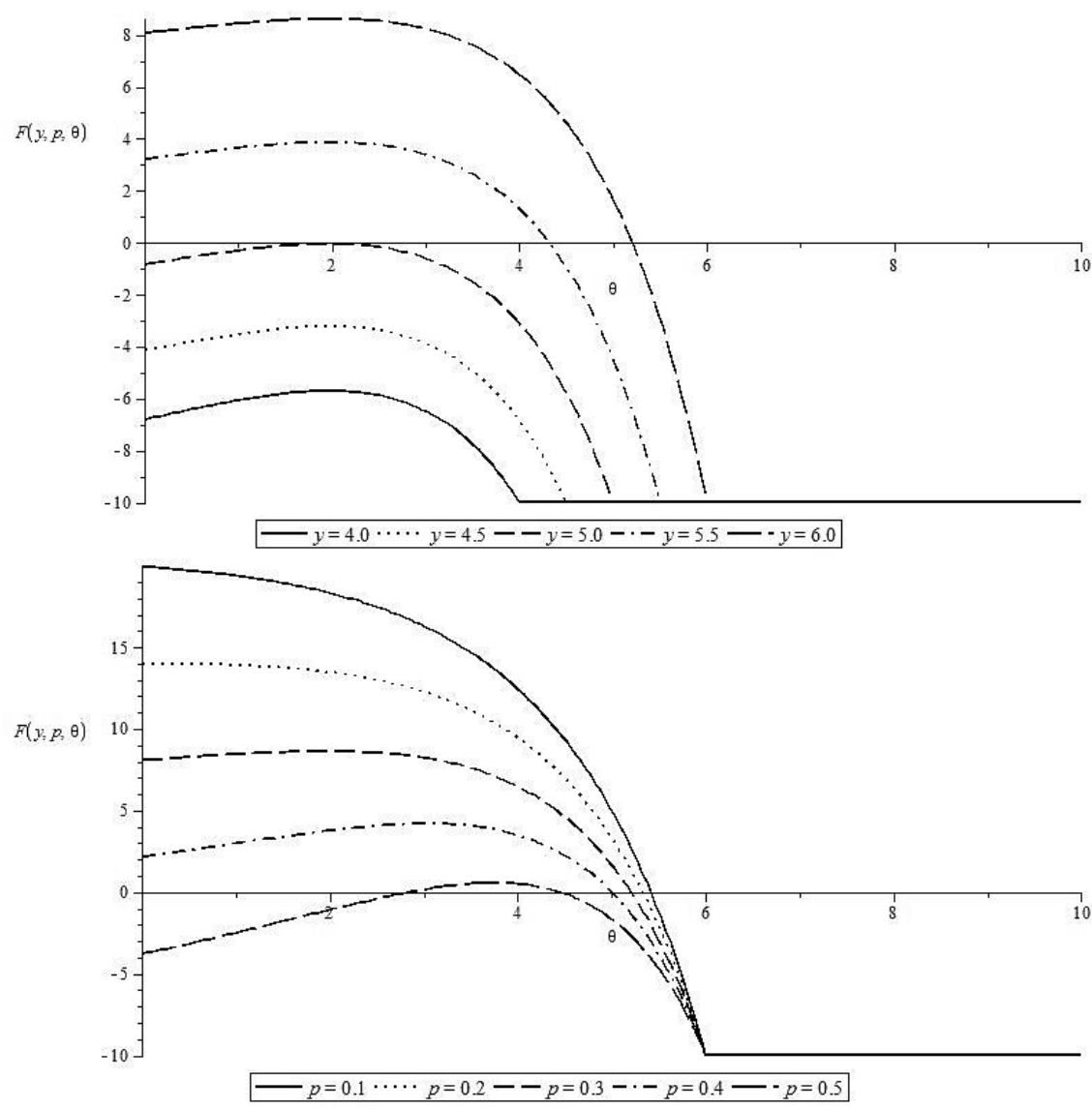

Fig. 5. The value of $F(y, p, \theta)$ with respect to $\theta$ for different levels of $y$ (upper) and $p$ (lower), for process $X$ with parameters $d=0.05, \sigma=0.4, \beta=0.1$ and with jumps from Erlang distribution $\operatorname{Erl}(2,4)$. Parameters $r=0.01, a=10, \alpha=100, c=10$, $p=0.3, y=6$

Source: own work. 


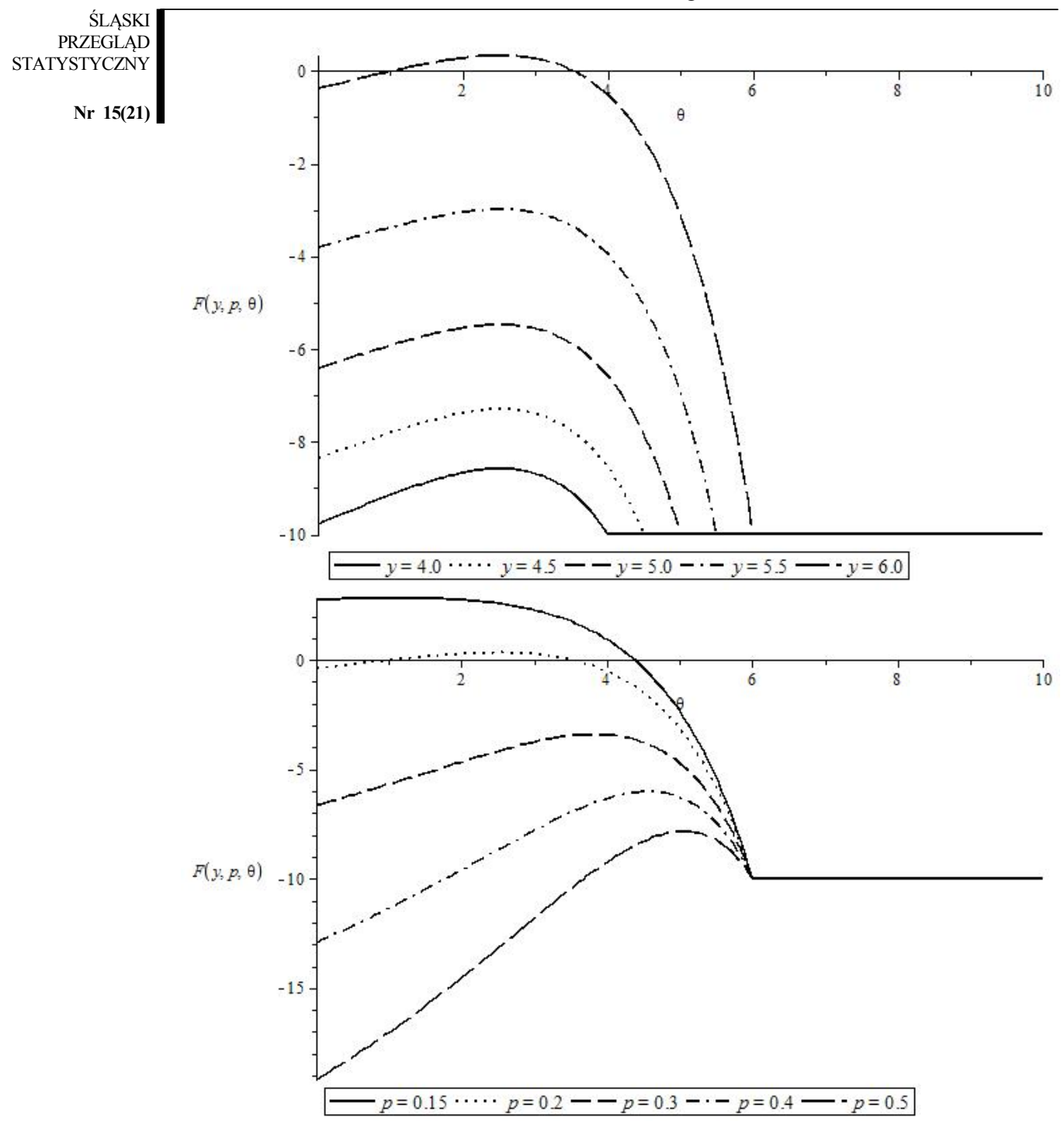

Fig. 6. The value of $F(y, p, \theta)$ with respect to $\theta$ for different levels of $y$ (upper) and $p$ (lower), for process $X$ with parameters $d=0.05, \sigma=0.4, \beta=0.1$ and with jumps from hyperexponential distribution $\gamma_{1}=0.4, \gamma_{2}=0.6, \rho_{1}=3, \rho_{2}=4$. Parameters $r=0.01, a=10, \alpha=100, c=10, p=0.2, y=6$

Source: own work.

increase $\theta^{*}$. Thus too high premium level produces contracts where $\theta^{*}>y$ and hence the insurance is no longer interesting for investors. On the other hand, we cannot decrease the premium rate too much sincondition (22) must be satisfied. If not, the optimal is to never 
terminate the contract earlier and maximum value is attained at 0 . For example, for jumps with Erlang distribution for our set of parameters this conditions means that the premium level should be strictly greater STATYSTYCZNY than 0.1 (see Figure 5 (lower) for $p=0.1$ ).

\subsection{Fair premium for drawup contingency}

We will now investigate numerically the insurance contract $k(y, z, p)$ given in (25) which provides protection from any specified drawdown of size $a$ with certain drawup contingency of size $a$. By Theorem 5 it suffices to find functions $\lambda$ and $v$. To do this we use identities (28)- (31). Therefore we can find that

$$
p^{*}=\frac{r \alpha\left(Z^{(r)}(a-y)-Z^{(r)}(2 a-y-z) \frac{W^{(r)}(a-y)}{W^{(r)}(2 a-y-z)}\right)}{1-Z^{(r)}(a-y)+\frac{W^{(r)}(a-y)}{W^{(r)}(2 a-y-z)}\left(Z^{(r)}(2 a-y-z)-1\right)}
$$

for $a \leq y+z$ and

$$
p^{*}=\frac{r \alpha\left(Z^{(r)}(z)-Z^{(r)}(a) \frac{W^{(r)}(a-y)}{W^{(r)}(a)}+\frac{1}{r} Z^{(r)}(a) \frac{W^{\prime(r)}(a)}{\left(W^{(r)}(a)\right)^{2}}\left(Z^{(r)}(a-y)-Z^{(r)}(z)\right)\right)}{1-Z^{(r)}(z)-\left(Z^{(r)}(a)-1\right)\left(\frac{1}{r} \frac{W^{\prime(r)}(a)}{\left(W^{(r)}(a)\right)^{2}}\left(Z^{(r)}(a-y)-Z^{(r)}(z)\right)\right)}
$$

for $a>y+z$.

Figure 7 and Figure 8 depict the value function for this contract. The solid line shows the dependency of contract value on the starting/historical position of drawdown $D_{0}=y$ with constant starting position of drawup $U_{0}=5$ and the dot line shows the dependency on starting position of drawup $z$ with constant starting position of drawdown $y=5$.

The dependency of fair premium $p^{*}$ with respect to starting position of drawdown and drawup was done on Figure 9 and Figure 10. Note that, similarly to the drawdown contract without drawup constraints, when $y \uparrow a$ the fair premium is growing to infinity since the drawdown of size $a$ will occur faster and the reward must be balanced by a premium, when it is fair. This is not the case when $z \uparrow$ $a$. Here the fair premium does not tend to infinity since there is no reward payment at $z=a$. 


\section{ŚLACSKI
PRZEGLĄD
STATYSTYCZNY
$\mathbf{N r} \mathbf{1 5 ( 2 1 )}$}

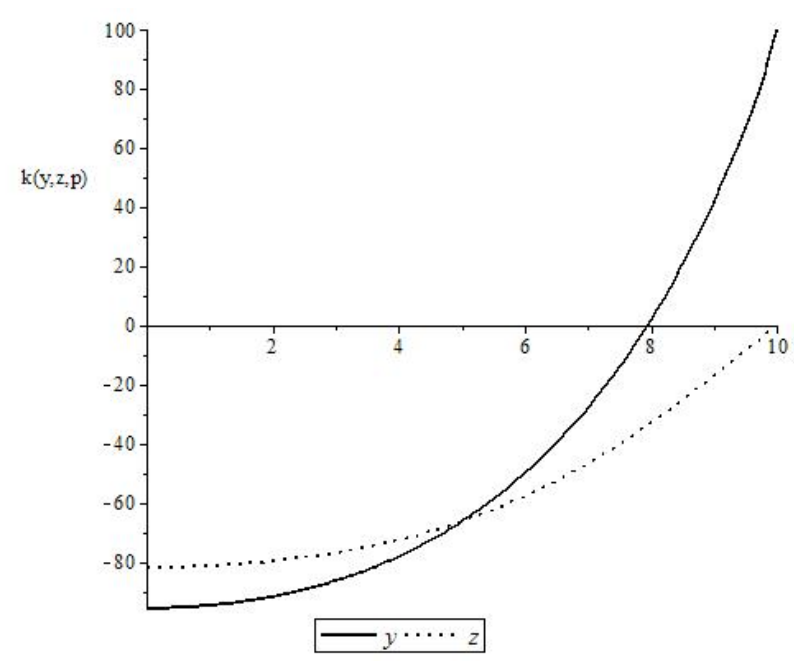

Fig. 7. The value of drawdown contract $k(y, z, p)$ with respect to $y$ with constant $z$ and with respect to $z$ with constant $y$, for process $X$ with parameters $d=0.05$, $\sigma=0.4, \beta=0.1$ and with jumps from Erlang distribution $\operatorname{Erl}(2,4)$. Parameters: $r=0.01, a=10, \alpha=100, p=1.4, y=5, z=5$

Source: own work.

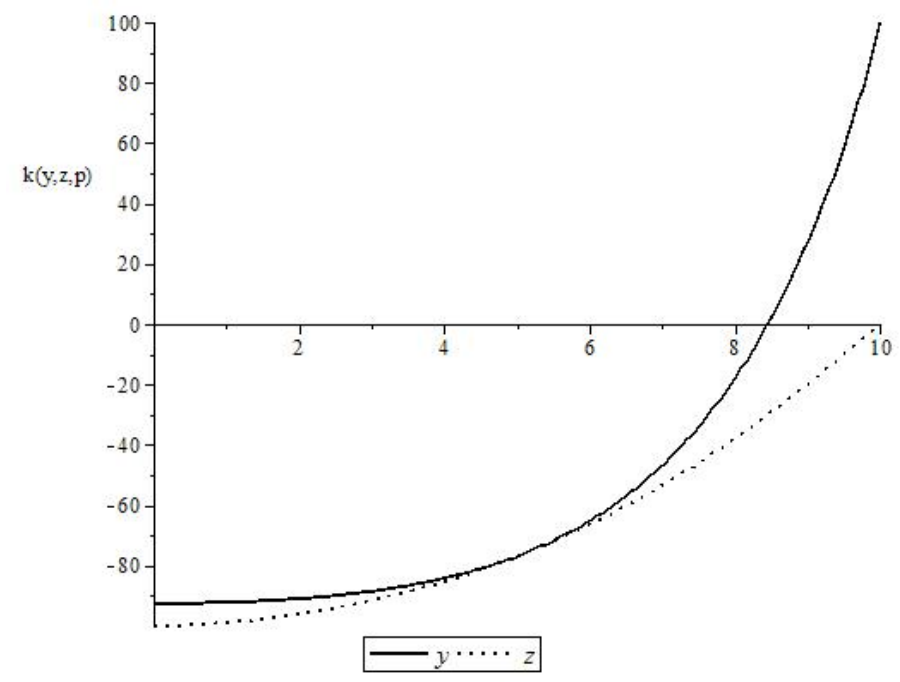

Fig. 8. The value of drawdown contract $k(y, z, p)$ with respect to $y$ with constant $z$ and with respect to $z$ with constant $y$, for process $X$ with parameters $d=0.05$, $\sigma=0.4, \beta=0.1$ and with jumps from hyperexponential distribution $\gamma_{1}=0.4, \gamma_{2}=0.6$, $\rho_{1}=3, \rho_{2}=4$. Parameters $r=0.01, a=10, \alpha=100, p=1.4, y=5, z=5$

Source: own work. 

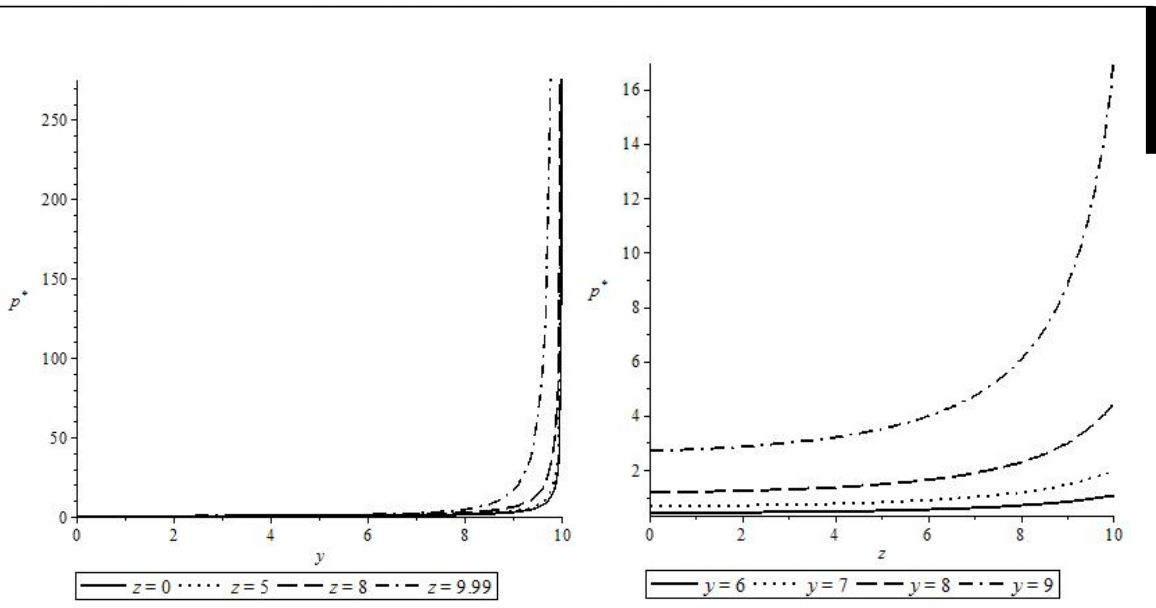

Fig. 9. Fair premium $p^{*}$ for drawup contingency contract $k(y, z, p)$ with respect to $y$ for different $z$ (upper) and with respect to $z$ for different $y$ (lower), for process $X$ with parameters $d=0.05, \sigma=0.4, \beta=0.1$ and with jumps from Erlang distribution $\operatorname{Erl}(2,4)$. Parameters: $r=0.01, a=10, \alpha=100, p=1.4$

Source: own work.
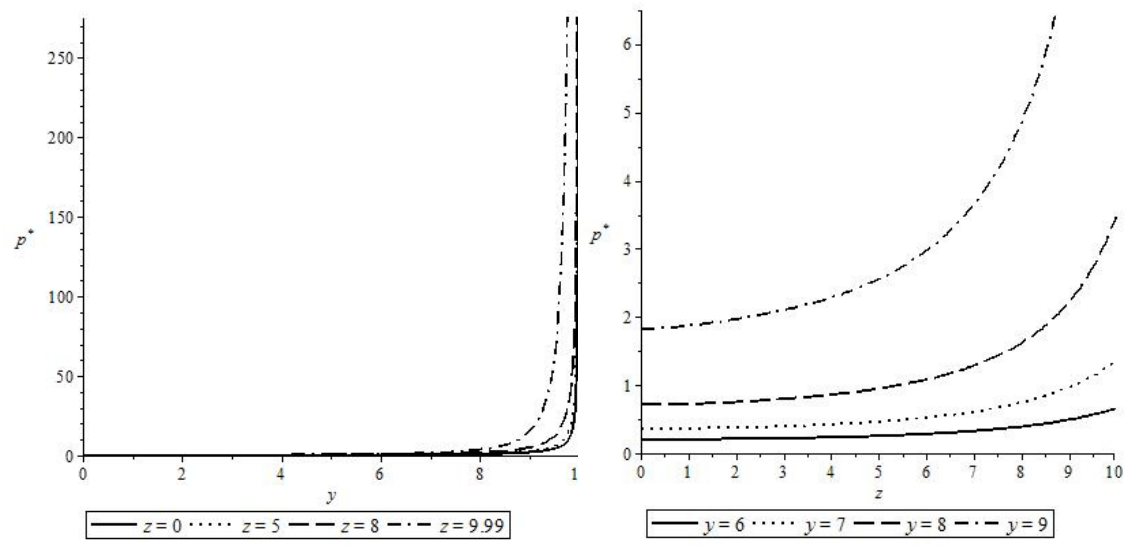

Fig. 10. Fair premium $p^{*}$ for drawup contingency contract $k(y, z, p)$ with respect to $y$ for different $z$ (upper) and with respect to $z$ for different $y$ (lower), for process $X$ with parameters $d=0.05, \sigma=0.4, \beta=0.1$ and with jumps from hyperexponential distribution $\gamma_{1}=0.4, \gamma_{2}=0.6, \rho_{1}=3, \rho_{2}=4$. Parameters $r=0.01, a=10, \alpha=100$, $p=1.4$

Source: own work. 


\subsection{Cancellable drawup contingency}

Nr 15(21)

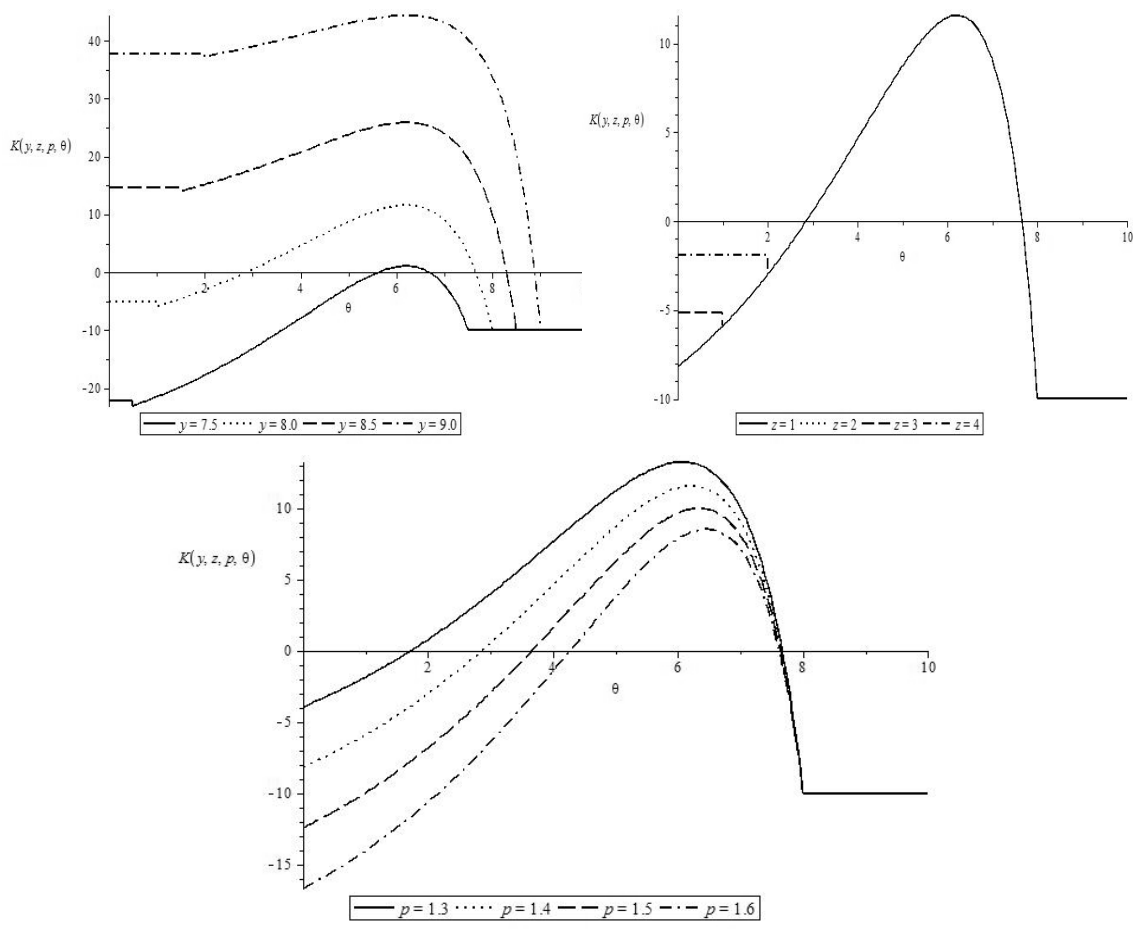

Fig. 11. The value of $K(y, z, p, \theta)$ with respect to $\theta$ for different levels of $y$ (left), $z$ (right) and $p$ (middle), for process $X$ with parameters $d=0.05, \sigma=0.4, \beta=0.1$ and with jumps from Erlang distribution $\operatorname{Erl}(2,4)$. Parameters $r=0.01, a=10, \alpha=100$, $c=10, p=1.4, y=8, z=2$

Source: own work.

We continue our previous numerical analysis by adding a cancellable feature and by considering the insurance contract (33). Note that by (37) it suffices to find the value of the contract without cancellable feature $k$ given in (25), hence, just to calculate functions $\lambda$ and $v$ given in (28)-(31) and scale functions (4) and (6).

The contract value is depicted in Figure 11 and Figure 12. What is worth mentioning is that in this contract there appears jump at $\theta=y+z-a$ if only $y+z-a>0$, which corresponds to the presence of a drawup contingency. This happens because when $\theta$ decreases the drawup increases and at $y+z-a$ we have a drawup of level $a$. The jump occurs since the contract terminates and the investor does not have to pay a fee. Furthermore, similarly to the previous case 
of drawdown contract with cancellable feature, we can observe that optimal $\theta^{*}$ does not depend on the starting position of drawdown $D_{0}=y$ and drawup $U_{0}=z$. What is more, the contract value depends STATYSTYCZNY on starting position of drawup $U_{0}=z$ only through the level when the jump appears. When there is no jump or if the jump has not appeared yet, the contract value for different $z$ coincides. However, the dependence is strong with respect to the process and contract parameters, like for example dependence of premium level $p$.

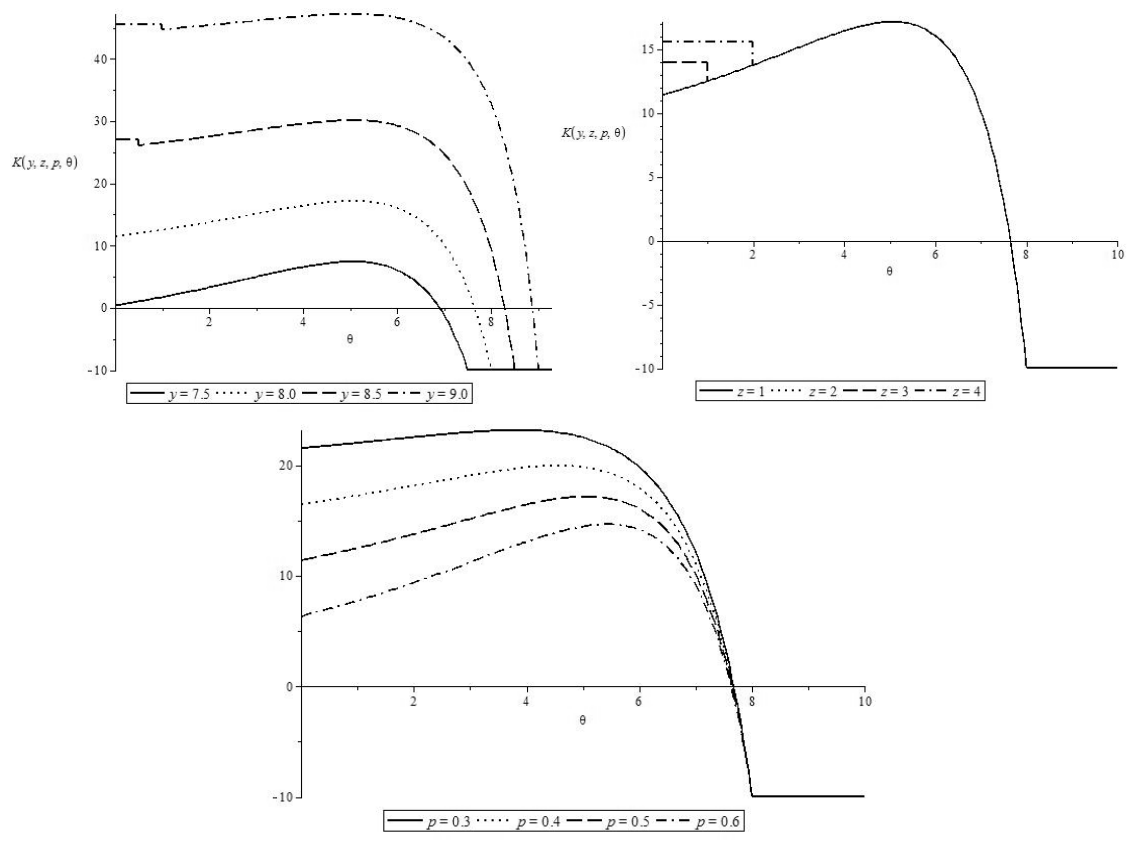

Fig. 12. The value of $K(y, z, p, \theta)$ with respect to $\theta$ for different levels of $y$ (left), $z$ (right) and $p$ (middle), for process $X$ with parameters $d=0.05, \sigma=0.4, \beta=0.1$ and with jumps from hyperexponential distribution $\gamma_{1}=0.4, \quad \gamma_{2}=0.6, \quad \rho_{1}=3, \rho_{2}=4$. Parameters $r=0.01, a=10, \alpha=100 c=10, p=0.5, y=8, z=2$

Source: own work.

\section{Conclusions}

In this paper we analyzed a few insurance contracts on drawdown and drawup events where the underlying asset is modeled by the CramérLundberg risk process perturbed by an independent Brownian motion $B_{t}$ and jumps given by phase-type distrbutions. We identified the fair premium $p^{*}$ and optimal stopping rules for the contracts with 
a cancelling feature. In this paper we focus on the crucial numerical analysis demonstrating the power of derived in [Palmowski, Tumilewicz 2016] theoretical formulas.

It is natural to consider other processes for asset prices. Moreover, one can consider fees $\alpha$ and $c$ dependent on the process $X$ observed at the end of the insurance contract. In fact, there is still a huge demand for more general insurance contracts that will serve as a policy against large drawdowns. This will be the subject of future research.

\section{References}

Asmussen S., 2003, Applied Probability and Queueus, Second edition. Springer-Verlag.

Carr P., Zhang H., Hadjiliadis O., 2011, Maximum drawdown insurance, International Journal of Theoretical and Applied Finance, 14(8), pp. 1-36.

Grossman S.J., Zhou Z., 1993, Optimal investment strategies for controlling drawdowns, Mathematical Finance, 3(3), pp. 241-276.

Egami M., Yamazaki K., 2014, Phase-type fitting of scale functions for spectrally negative Lévy processes, J. Comput. Appl. Math., 264, pp. 1-22.

Kyprianou A.E., 2006, Introductory Lectures on Fluctuations of Lévy Processes with Applications, Springer.

Kyprianou A.E, Kuznetsov A., Rivero V., 2013, The Theory of Scale Functions for Spectrally Negative Lévy Processes, Lévy Matters II, Springer Lecture Notes in Mathematics.

Magdon-Ismail M., Atiya A., 2004, Maximum drawdown, Risk, 17(10), pp. 99-102.

Mijatović A., Pistorius M.R., 2012, On the drawdown of completely asymmetric Lévy process, Stochastic Processes and their Applications, 122(11), pp. 3812-3836.

Nguyen-Ngoc L., Yor M., 2005, Some Martingales Associated to Reflected Lévy Processes, Séminaire de Probabilités, XXXVIII, pp. 42-69.

Oksendal B., Sulem A., 2004, Applied Stochastic Control of Jump Diffusions, Springer.

Palmowski Z., Tumilewicz J., 2016, Pricing insurance drawdown-type contracts with underlying Lévy assets, submitted for publication.

Peskir G., Shiryaev A., 2006, Optimal Stopping and Free-Boundary Problems, Birhäuser.

Pistorius M.R., 2004, On exit and ergodicity of the spectrally one-sided Lévy process reflected at its infimum, Journal of Theoretical Probability, 17(1), pp. 183-220.

Pospisil L., Vecer J., 2010, Portfolio sensitivities to the changes in the maximum and the maximum drawdown, Quantitative Finance, 10(6), pp. 617-627.

Sornette D., 2003, Why Stock Markets Crash: Critical Events in Complex Financial Systems, Princeton University Press.

Vecer J., 2006, Maximum drawdown and directional trading, Risk, 19(12), pp. 88-92.

Vecer J., 2007, Preventing portfolio losses by hedging maximum drawdown, Wilmott, 5(4) pp. $1-8$.

Zhang H., Hadjiliadis O., 2010, Drawdowns and rallies in finite time-horizon, Methodology and Computing in Applied Probability, 12(2), pp. 293-308.

Zhang H., Leung T., Hadjiliadis O., 2013, Stochastic modeling and fair valuation of drawdown insurance, Insurance: Mathematics and Economics, 53(2013), pp. 840-850. 


\section{SPADKI I WZROSTY FAZOWYCH PROCESÓW LÉVY'EGO W WYCENIE KONTRAKTÓW UBEZPIECZENIOWYCH}

Streszczenie: W artykule rozważamy kontrakty ubezpieczeniowe oparte na procesach spadku i wzrostu dla procesów ryzyka typu Lévy'ego z fazowymi stratami. Proces spadku/wzrostu w danej chwili określamy jako różnicę pomiędzy supremum/infimum do tego momentu $\mathrm{i}$ wartością procesu $\mathrm{w}$ tej chwili. W pracy przeanalizujemy cztery kontrakty. Pierwszy kontrakt zobowiązuje inwestora do płacenia stałej składki w sposób ciągły, a w zamian - w momencie, gdy proces spadku przekroczy określony poziom wypłaca inwestorowi określone wynagrodzenie. Drugi kontrakt może zostać samoistnie rozwiązany w chwili zaobserwowania procesu wzrostu do pewnego poziomu. Ostatnie dwa kontrakty dają inwestorowi dodatkowo możliwość wcześniejszego wycofania się z umowy po uiszczeniu z góry ustalonej grzywny. Praca opiera się na wcześniejszym artykule [Palmowski, Tumilewicz 2016] i koncentruje się na statystycznym aspekcie wcześniej otrzymanych formuł, modelując skoki cen akcji poprzez rozkłady fazowe.

Słowa kluczowe: proces spadku, proces wzrostu, procesy Lévy'ego, kontrakty ubezpieczeniowe, proces fazowy. 